\title{
Oxidative stress and antioxidant defences - a pedagogical review
}

\author{
Stress oxidativo e defesas antioxidantes - uma revisão pedagógica
}

\author{
Ana Sofia Fernandes ${ }^{1,2}$, Matilde Castro ${ }^{1}$, Nuno Guerreiro Oliveira ${ }^{1}$ \\ 1 Research Institute for Medicines and Pharmaceutical Sciences (iMed.UL), Faculdade de Farmácia, \\ Universidade de Lisboa, Av. Prof. Gama Pinto, 1649-003 Lisboa, Portugal \\ 2 Centro de Investigação em Cências e Tecnologias da Saúde (C BIOS) Universidade Lusófona, \\ Campo Grande 376, 1749-024 Lisboa, Portugal \\ E-mail: anasofiafemandes@iff.ul.pt
}

\begin{abstract}
Abstrac
tIn the lastyears a growing interest concerning the effects of oxidativestress has been noticed and its involvement in different pathological phenomena suggested by many scientific evidences. The present paper aims to review oxidative stress basic concepts and antioxidation, introducing health professionals and students to this topic. Thus, main reactive oxygen species generation pathways and lipid peroxidation processes are addressed under this view. This paper focus antioxidation defenses, superoxide dismutases, catal ase and glutathione peroxidases in particular, enzymes playing a pivotal role in this cellular context. The present review also mentions the oxidative stress deleterious effects and pathologies wher oxidative damage is involved, justifying the interest of antioxidative compounds.
\end{abstract}

Keywords: oxidative stress, reactive oxygen species, antioxidant defenses

\begin{abstract}
Resumo
Nos últimos anos tem havido um interesse crescentepelos efeitos do stress oxidativo, emuitas evidências científicas indicam que este está envolvido em diversos fenómenos patológicos. O presente artigo pretende rever os conceitos de stress oxidativo e defesas antioxidantes, permitindo introduzir os profissionais e alunos da área da saúde nesta temática. Assim, são abordadas as principais vias de formação de espécies reactivas de oxigénio, assim como o processo de peroxidação lipidica. Este artigo foca também as defesas antioxidantes, especialmente as enzimas superóxido dismutases, catalasee glutationo peroxidases, que desempenham um papel central nas defesas celulares. São ainda referidos os efeitos deletérios do stress oxidativo, bem como alguns dos processos patológicos em que as lesões oxidativas estão envolvidas, eque justificam o interesse dos compostos antioxidantes.
\end{abstract}

Palavras-chave: stress oxidativo, espécies reactivas deoxigénio, defesas antioxidantes 


\section{Introduction}

In the last years a growing interest around the oxidative stress effects has been noticed. In fact, many scientific evidences points to the oxidative stress involvement in various pathologies (e.g. cancer, inflammatory disorders, cardiovascular, pulmonary and neurodegenerative diseases), as in the toxicity mechanism of xenobiotics, or even in the ageing process. Consequently, many consumer products are enriched with antioxidants, as it happens with food (e.g. juice, cookies), cosmetics (c.g. anti-ageing products, sunscreens) or nutritional supplements. This paper aims to explain the oxidative stress and antioxidants concepts, introducing health professionals and students to this emerging field.

The term "oxidative stress" may be defined as an imbalance between oxidants and antioxidants favouring the oxidants, potentially leading to damage . Oxidants are formed as regular aerobic metabolism products and, under physiological conditions, their production is balanced by the antioxidant defences . However, if the production of oxidants increases or antioxidant defences decreases, oxidative damage occurs.

\section{Reactive oxygen species}

The collective term reactive species (RS) includes oxygen, nitrogen, chlorine, bromine and sulphur transient species with high chemical reactivity. These include free radicals, i.e., species containing one or more unpaired electrons, and non-radical derivatives . Reactive oxygen species (ROS) encompasses a variety of diverse chemical species including superoxide anion $(\mathrm{O} 2-)$, hydroxyl radical $(\mathrm{HO})$, hydrogen peroxide ( $\mathrm{H} 2 \mathrm{O} 2)$, singlet oxygen (1O2), alkoxyl radicals (RO), and peroxyl radicals (ROO). Some ROS, such as $\mathrm{O}_{2}-$ or $\mathrm{HO}$, are extremely unstable and reactive. In contrast, other $\mathrm{ROS}$ like $\mathrm{H} 2 \mathrm{O} 2$ or $\mathrm{ROO}$ are relatively more stable, with half-lives in the range of seconds. These species may diffuse away from their site of generation, transporting the radical or oxidant function to other target sites. Reactive nitrogen species, such as peroxynitrite (ONOO-), are also relevant in numerous pathophysiological phenomena. Fig. 1 shows the generation pathways of ROS. Superoxide anion is formed in biological systems by the partial reduction of molecular oxygen. Reduction of $\mathrm{O} 2$ - with a second electron, as well as a two-electron reduction of $\mathrm{O} 2$, generates $\mathrm{O} 22-$, which leads to $\mathrm{H} 2 \mathrm{O} 2$. The oneelectron reduction of $\mathrm{H} 2 \mathrm{O} 2$, that occurs in the presence of reduced transition metals like $\mathrm{Cu}(\mathrm{I})$ and $\mathrm{Fe}(\mathrm{II})$, originates HO. Other reactive species can be generated by the reaction of ROS with biological molecules (e.g.

\section{Introduçäo}

Nos últimos anos tem havido um interesse crescente pelos efeitos do stress oxidativo. De facto, muitas evidencias científicas indicam que o stress oxidativo está envolvido em diversas patologias (ex. cancro, doenças inflamatórias, cardio-vasculares, pulmonares e neurodegenerativas), assim como nos mecanismos de toxicidade de muitos xenobióticos e no processo de envelhecimento. Consequentemente, muitos produtos de consumo corrente contêm antioxidantes na sua formulação, como é o caso de alguns produtos alimentares (ex. sumos, bolachas), cosméticos (ex. produtos anti-envelhecimento, protectores solares) ou suplementos alimentares. Este artigo pretende abordar os conceitos de stress oxidativo e antioxidantes, permitindo introduzir os profissionais e alunos da área da saúde nesta área de interesse crescente.

$O$ stress oxidativo pode definir-se como uma perturbação do equilibrio entre oxidantes e antioxidantes, a favor dos oxidantes, podendo levar à ocorrência de lesões nas células, tecidos ou órgãos [1]. As espécies oxidantes formam-se como produtos naturais do metabolismo aeróbio e, em condições fisiológicas, a sua produção é compensada pelas defesas antioxidantes [2]. No entanto, se a produção de oxidantes aumentar ou as defesas antioxidantes diminuirem, podem ocorrer lesões oxidativas [3].

\section{Espécies reactivas de oxigénio}

O termo espécies reactivas (ER) inclui espécies transientes de oxigénio, azoto, cloro, bromo ou enxofre, com elevada reactividade quimica ${ }^{12,4]}$. Estão incluidas espécies radicalares, i.e., espécies com um ou mais electrōes desemparelhados, assim como espécies não radicalares ${ }^{[3]}$ As espécies reactivas de oxigénio (ERO) incluem o aniäo superóxido $\left(\mathrm{O}_{2}-\right)$, radical hidroxilo (HO), peróxido de hidrogénio $\left(\mathrm{H}_{2} \mathrm{O}_{2}\right)$, oxigénio singleto $\left(\mathrm{O}_{2}\right)$, radicais alcoxilo $(\mathrm{RO})$ e radicais peroxilo (ROO) ${ }^{[2,4]}$. Algumas ERO, como o $\mathrm{O} 2$ - ou o $\mathrm{HO}$, são extremamente instáveis e reactivas. No entanto, outras ERO como $\mathrm{H}_{2} \mathrm{O}_{2}$ ou ROO são relativamente mais estáveis, com tempos de semi-vida na ordem dos segundos. Estas espécies podem difundir do seu local de formação, transportando as funções radicalares e oxidantes para outros locais alvo ${ }^{[1,5]}$. Para além das ERO, também as espécies reactivas de azoto, tais como o peroxinitrito (ONOO-), são importantes em diversos fenómenos fisiopatológicos ${ }^{[26.7]}$. Na Fig. 1 estão representadas as vias de formação de ERO. O anião superóxido forma-se nos sistemas biológicos através da redução parcial do oxigénio molecular. A redução do $\mathrm{O}_{2}-$ com um segundo electrão, assim como a redução de $\mathrm{O}_{2}$ por dois electrôes, gera $\mathrm{O} 22$-, que leva à formação de $\mathrm{H} 2 \mathrm{O} 2$. A redução do $\mathrm{H} 2 \mathrm{O} 2$, que é 
polyunsaturated lipids, thiols and nitric oxide (NO)) For example, the reaction between $\mathrm{O}_{2}-$ and $\mathrm{NO}$ originates $\mathrm{ONOO}$-, which is unstable at physiological $\mathrm{pH}$ and rapidly decomposes to form potent nitrating and oxidizing species. Other species resulting from the RS reaction with biomolecules are radicals alkoxyl and peroxyl. These can be generated by a variety of routes, including the reaction of lipid peroxides with $\mathrm{HO} 2$, the breakdown of organic peroxides, and the reaction of RS with lipids or amino acid radicals . facilitada pela presença de metais de transição reduzidos como $\mathrm{Cu}(\mathrm{I})$ ou $\mathrm{Fe}$ (II), origina $\mathrm{HO}^{[3.10]}$. Outras espécies reactivas podem ser formadas pela reacçāo de ERO com moléculas biológicas (ex. lípidos polinsaturados, tiois e monóxido de azoto $(\mathrm{NO}))^{\mathrm{Dm}}$. Por exemplo, a reacção entre $\mathrm{O}_{2}-$ e NO origina $\mathrm{ONOO}-, \mathrm{o}$ qual é instável a $\mathrm{pH}$ físiológico decompondo-se rapidamente para originar espécies fortemente nitrantes e oxidantes ${ }^{[2,10]}$. Outras espécies que resultam da reaç̧ão de ER com biomoléculas são os radicais alcoxilo e peroxilo, que podem ser formados por várias vias incluindo a reacção de lípidos com $\mathrm{HO}_{2}$, a degradação de peróxidos orgânicos, ou a reacção de ER com radicais derivados de lípidos ou aminoácidos ${ }^{[2.1]}$.

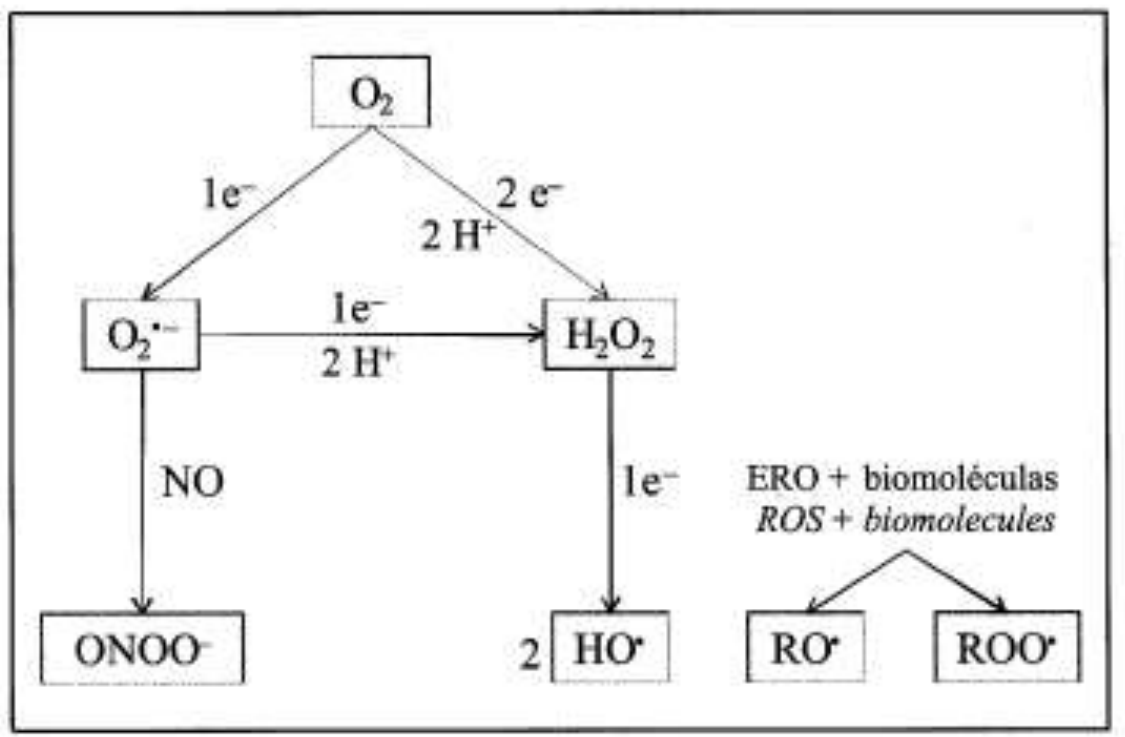

Figure 1 - Generation of the most relevant reactive oxygen species (adapted from ${ }^{110}$ ). Figura 1 - Formação das principais espécies reactivas de oxigénio (adaptado de ${ }^{\text {tho }}$ ).

ROS can be generated as a result of normal cellular metabolism. The majority of intracellular ROS production results from the mitochondria . In fact, $\mathrm{O}_{2}$ is formed from the uncoupling of the mitochondrial electron transport chain during oxidative phosphorylation, Also, the catalytic action of a variety of intracellular and extracellular oxidases like NADPH oxidase, xanthine oxidase or lipooxygenase, as well as the metabolism of arachidonic acid, gives rise to $\mathrm{O}_{2}-$. Superoxide can also be produced by the phagocytic NADPH oxidase, during host defence responses, where $\mathrm{O} 2-$ is thought to act in cell-signalling and killing foreign bacteria . Other ROS are also generated in peroxisomes, as well as from a variety of cytosolic enzyme systems. Another closely related process of
As ERO podem ser geradas como resultado do metabolismo celular normal, sendo que a maioria das ERO intracelulares são produzidas na mitocôndria ${ }^{[3]}$. De facto, o O2 - é gerado na cadeia de transporte de

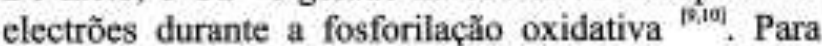
além desta via, o $\mathrm{O} 2$ - pode ter origem na acçăo catalitica de várias oxidases intra e extracelulares, tais como a NADPH oxidase, xantina oxidase ou lipooxigenase, assim como também no metabolismo do ácido araquidónico ${ }^{[\mathrm{l} !}$. $\mathrm{O}_{2} \mathrm{O}_{2}-$ pode também ser formado pela NADPH oxidase dos fagócitos durante a resposta imunitária, onde $\mathrm{o}_{2}-$ parece actuar na sinalização celular e na eliminação de bactérias ए, ए., Outras ERO podem ter origem nos peroxissomas, assim como também em diferentes enzimas 
ROS formation is the lipid peroxidation (LPO), i.e., the oxidative deterioration of polyunsaturated lipids (Fig. 2) . LPO is initiated by the addition of a RS (e.g. $\mathrm{HO}, \mathrm{HO} 2$, $\mathrm{RO}, \mathrm{ROO}$ ) to an unsaturated lipid, or by the hydrogen subtraction from a methylene group executed by a RS, producing a carbon radical. Carbon radicals often stabilize by molecular rearrangement, generating conjugated dienes. Carbon radicals can also react with O2, giving rise to lipid peroxyl radicals (LOO). These radicals can abstract $\mathrm{H}$ from an adjacent lipid, propagating the process. The combination of $\mathrm{LOO}$ with $\mathrm{H}$ generates a lipid hydroperoxide (LOOH). The decomposition of $\mathrm{LOOH}$ in the presence of transition metal ions produces lipid alkoxyl radicals (LO) that may also abstract $H$ from unsaturated lipids, continuing the propagation of LPO. These phenomena may occur in biological membranes, leading to oxidative damage. In fact, the continued oxidation of fatty-acid side chains and their fragmentation to produce aldehydes and hydrocarbons may lead to the loss of membrane integrity. In addition, products of lipid peroxidation (c.g. isoprostanes, malondialdehyde, 4-hydroxynonenal) may exert by themselves, deleterious effects, namely DNA damage and proteins inhibition, leading to different phenomena including cytotoxicity, allergy, mutagenicity, and carcinogenesis. citosólicas $^{[59]}$. Um outro processo intimamente ligado à formaçăo de ERO é a peroxidaçåo lipidica, i.e., a degradação oxidativa de lipidos polinsaturados (Fig, 2) ${ }^{|2|}$. A peroxidação lipidica inicia-se por adição de uma ER (ex. $\mathrm{HO}, \mathrm{HO} 2, \mathrm{RO}, \mathrm{ROO}$ ) a um lípido insaturado, ou por remoção de um hidrogénio de um grupo metileno por uma ER, originando um radical centrado no carbono. Estes radicais estabilizam frequentemente por rearranjo molecular, formañndo dienos conjugados. Os radicais centrados no ćarbono podem também reagir com o oxigénio, dando origem a radicais peroxilo lipídicos (LOO). Estes podem remover um $\mathrm{H}$ de um lipido adjacente, propagando o processo. Por outro lado, a combinação de LOO com $\mathrm{H}$ origina hidroperóxidos lipidicos ( $\mathrm{LOOH}$ ). A decomposiçăo de $\mathrm{LOOH}$ na presença de metais de transição produz radicais alcoxilo lipidicos (LO), os quais também podem remover $\mathrm{H}$ de lípidos insaturados e propagar o processo de peroxidação lipídica. Este fenómeno pode ocorrer em membranas biológicas, levando a lesões oxidativas. De facto, a oxidação continuada das cadeias laterais dos ácidos gordos e a sua fragmentação para produzir aldeídos e hidrocarbonetos pode resultar na perda de integridade membranar. Adicionalmente, os próprios produtos da peroxidação lipidica (ex. isoprostanos, malondialdeido, 4-hidroxinonenal) podem exercer efeitos deletérios ${ }^{(1)}$, nomeadamente lesỏes no $\mathrm{ADN} \mathrm{e}$ inibição de proteinas, levando a fenómenos de citotoxícidade, alergia, mutagenicidade e cancerigénese $\mathrm{e}^{[12]}$.
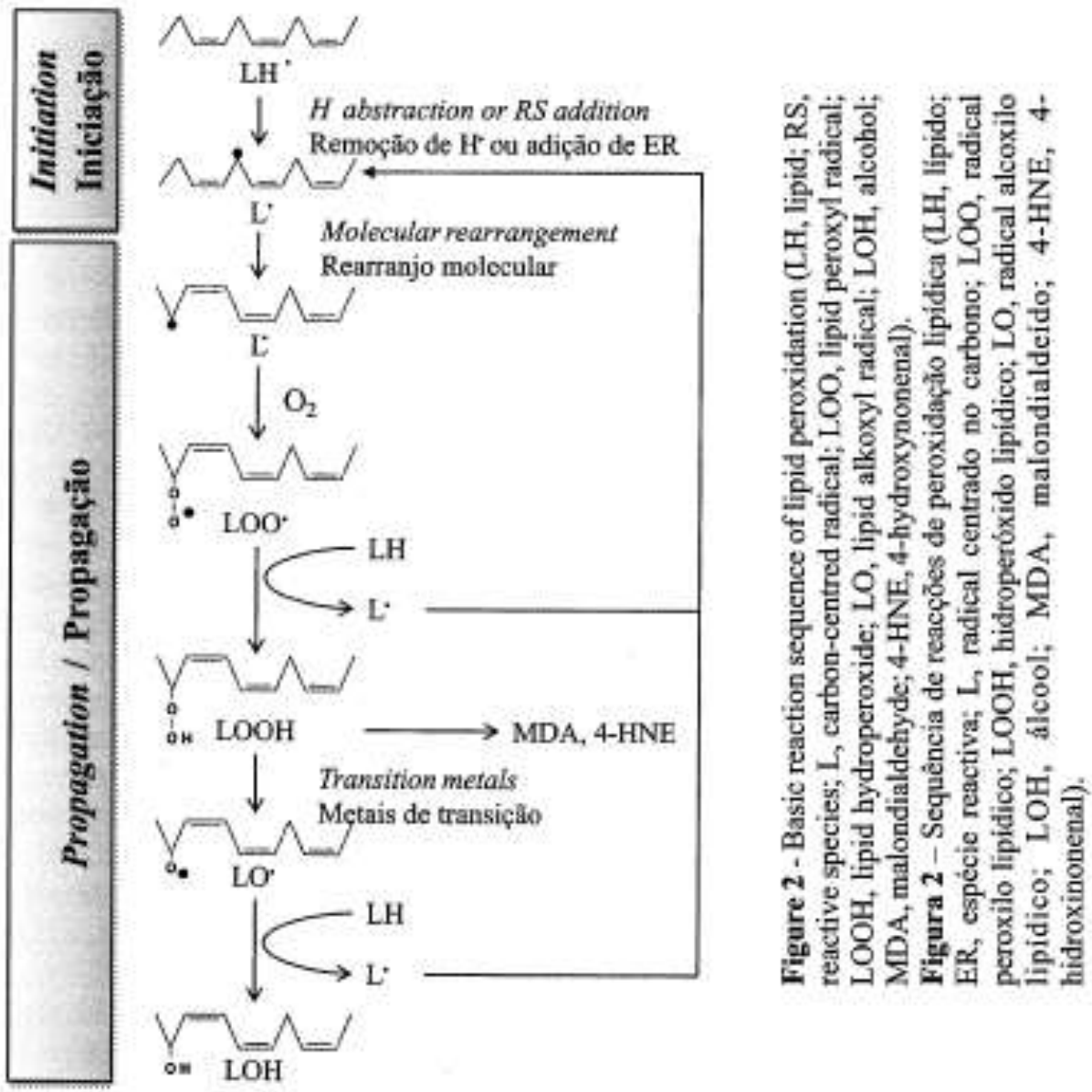
Despite the endogenous sources of ROS, a number of external agents can trigger ROS production. Different types of radiation, such as ultraviolet, ultrasound, microwave, and ionizing radiation (e.g. X-and $\square$-rays) are known to generate ROS. Also, some chemotherapeutic agents (e.g. doxorubicin, bleomycin), some antibiotics (e.g. tetracyclines, aminoglycosides), heavy metals, herbicides, atmospheric pollutants, and environmental toxins can shift cells into a state of oxidative stress .

Although high concentrations of ROS may cause oxidative damage, low concentrations of ROS are needed to regulate several key physiological processes. These include cell differentiation, cell proliferation and apoptosis, regulated by redox-sensitive signal transduction pathways.

\section{Antioxidant defences}

Under physiological conditions, the ROS production is counteracted by an intricate antioxidant defence system . An antioxidant has been defined as a substance that significantly delays or prevents oxidation of a substrate, even in low concentrations when compared with these of an oxidizable substrate. More recently, Halliwell and Gutteridge altered this definition in order to account chaperones, repair systems and inhibitors of RS generation. Presently, an antioxidant is defined as any substance that delays, prevents or removes oxidative damage in a target molecule. This concept includes non-enzymatic compounds, as well as antioxidant enzymes. Non-enzymatic antioxidant defences comprises a number of low molecular weight molecules with the ability to scavenge ROS. These include compounds synthesized in vivo (e.g. glutathione, bilirubin, pyruvate, melatonin, coenzyme Q, uric acid), as well as agents obtained from the diet (e.g. ascorbate, tocopherol, carotenoids, flavonoids) . Eukaryotic cells possess an efficient antioxidant enzymatic network, as depicted in Fig. 3. Three major classes of antioxidant enzymes includes superoxide dismutases (SOD), catalases (CAT) and glutathione peroxidases (GPx), which will be reviewed latter on.
Para além das fontes endógenas de ERO, há também numerosos agentes extemos que provocam o aumento destas espécies ${ }^{19}$ Diferentes tipos de radiaçăo, como a radiação ultravioleta, ultrasons, microondas e radiação ionizante (ex. raios X e y) originam ERO ${ }^{1291}$, Outros agentes que levam a stress oxidativo são alguns fármacos antitumorais (ex. doxorrubicina, bleomicina), alguns antibióticos (ex. tetraciclinas, aminoglicosidos), metais pesados, herbicidas, poluentes atmosféricos e toxinas ambientais ${ }^{12,5}$.

Apesar das ERO, em concentraçōes elevadas, causarem lesões oxidativas, em concentraçōes baixas podem exercer funções reguladoras em vários processos fisiológicos essenciais ${ }^{[0,9,15]}$ É o caso da diferenciação celular, proliferação e apoptose que dependem de mecanismos de transduçâo de sinal susceptíveis de sofrer regulação redox. . $^{10,0,13]}$

\section{Defesas antioxidantes}

Em circunstâncias fisiológicas, a formação de ERO é compensada por um sistema complexo de defesas antioxidantes ${ }^{13, s]}$. Um antioxidante pode ser definido como uma substância que, quando presente $\mathrm{em}$ concentrações baixas comparativamente as de um substracto oxidável, retarda significativamente ou previne a oxidação desse substracto ${ }^{[2,3,1]}$. Recentemente, Halliwell e Gutteridge ${ }^{\mathrm{II}}$ alteraram esta definição de modo a incorporar proteinas chaperones, sistemas de reparação e inibidores da formação de ERO. Assim, um antioxidante é actualmente definido como uma substância que retarda, previne ou remove lesões oxidativas de uma molécula alvo ${ }^{[2 /}$ Este conceito inclui compostos não enzimáticos, assim como também enzimas antioxidantes ${ }^{[1]}$. As defesas antioxidantes não enzimáticas incluem uma grande variedade de moléculas com a capacidade de sequestrar ERO ${ }^{[x, 9]}$, incluindo compostos sintetizados in vivo (ex. glutationo, bilirrubina, piruvato, melatonina, coenzima $\mathrm{Q}$, ácido úrico), assim como substàncias provenientes da dieta (ex. ascorbato, tocoferol, carotenoides, flavonóides) ${ }^{[2]}$. As células eucarióticas possuem uma rede eficaz de enzimas antioxidantes, como ilustrado na Fig. 3, sendo que as principais enzimas são as superóxido dismutases (SOD), catalases (CAT) e glutationo peroxidases $(\mathrm{GPx})^{\text {"1) }}$, as quais serão descritas mais detalhadamente neste artigo. 


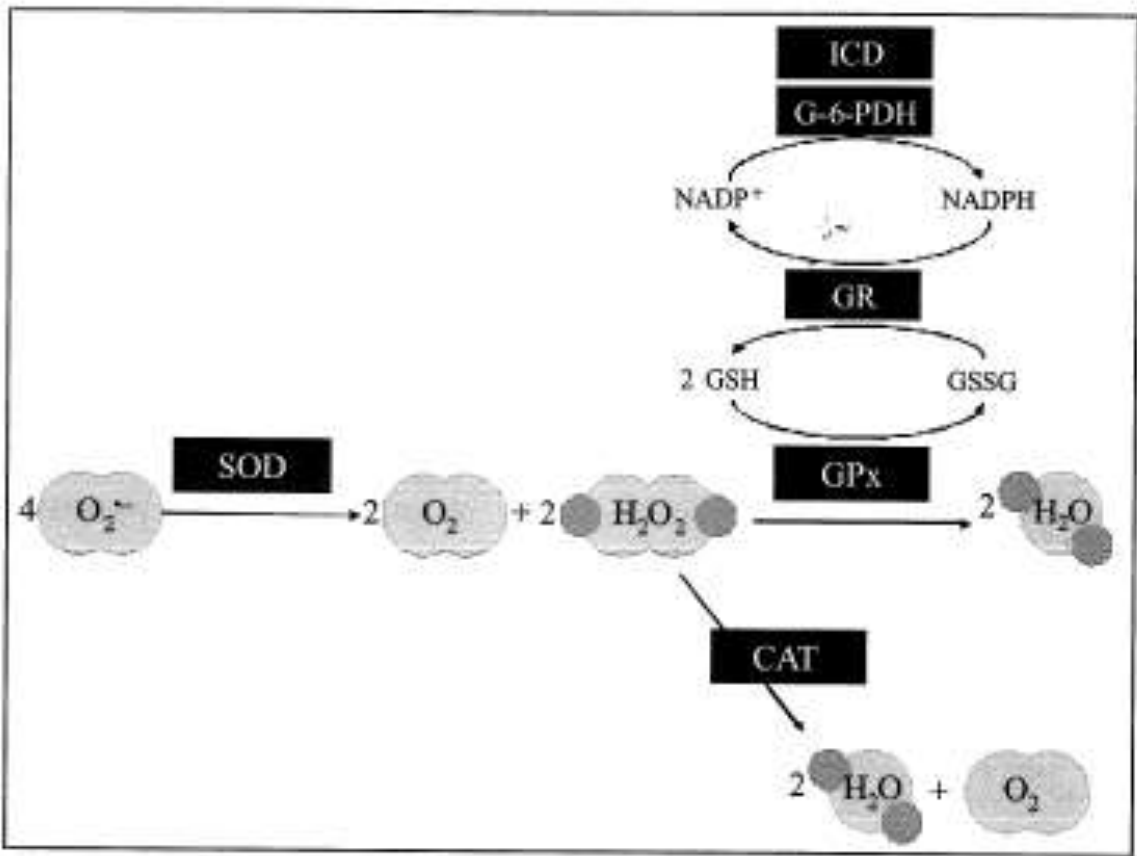

Figure 3 - Enzymatic antioxidant defences: role of superoxide dismutases (SOD), catalases (CAT), glutathione peroxidases (GPx), glutathione reductase (GR), glucose-6-phosphate dehydrogenase (G-6-PDH), and NADP+-dependent isocitrate dehydrogenase (ICD) on ROS detoxification (adapted from $[8,13]$ ).

Figura 3 - Defesas antioxidants enzimáticas: papel das superóxido dismutases (SOD), catalases (CAT), glutationo peroxidases (GPx), glutationo reductase (GR), glucose-6-fosfato desidrogenase (G-6-PDH), e isocitrato desidrogenase NADP+dependente (ICD) na destoxificação de espécies reactivas de oxigénio (adaptado de $[8,13])$.

\section{Superoxide dismutase}

Superoxide dismutases, whose enzymatic activity was discovered in 1969 by McCord and Fridovich, are metalloproteins with oxido-reductase capacity that catalyze the $\mathrm{O} 2$ - dismutation into oxygen and $\mathrm{H}_{2} \mathrm{O} 2$, according to the following equations:

\section{Superóxido dismutases}

As superóxido dismutases, cuja actividade enzimática foi descoberta em 1969 por McCord e Fridovich, são metaloproteinas com capacidade oxidoredutásica, que catalizam a reaç̧ão de dismutação do $\mathrm{O}_{2}-\mathrm{em} \mathrm{H}_{3} \mathrm{O}_{2} \mathrm{eO}_{2}$ ${ }^{[2, L]}$ de acordo com as seguintes equações:

$$
\begin{aligned}
& \mathrm{O}_{2}^{--}+\mathrm{M}^{(\mathrm{n}+1)+} \rightarrow \mathrm{O}_{2}+\mathrm{M}^{\mathrm{at}} \\
& \underline{\mathrm{O}}_{2}^{--}+\mathrm{M}^{\mathrm{n+}}+2 \mathrm{H}^{+} \rightarrow \mathrm{H}_{2} \mathrm{O}_{2}+\mathrm{M}^{(\mathrm{n}+1)+}
\end{aligned}
$$

Net reaction / Reacção Global : $2 \mathrm{O}_{2}^{-} \rightarrow \mathrm{O}_{2}+\mathrm{H}_{2} \mathrm{O}_{2}$ 
The oxidized form of the metalloenzyme SOD $(\mathrm{M}(\mathrm{n}+1)+)$ reacts with one $\mathrm{O} 2$ - to form $\mathrm{O} 2$ and generates the enzyme's (Mn+) reduced form (Eq 1). In the second step of the dismutation reaction, the reduced enzyme reacts with another $\mathrm{O} 2$ - and two protons to form $\mathrm{H} 2 \mathrm{O} 2$, regenerating the enzyme's oxidized form (Eq 2). Three SOD isoforms with different characteristics have been characterized in mammals (Table 1). Further classes of SOD have been identified, namely nickel-containing SODs in Streptomyces sp. and some cyanobacteria, and iron-containing SODs in bacteria, algae, trypanosomes and higher plants .
A metaloenzima na sua forma oxidada $\left(\mathrm{M}^{\left(\mathrm{in}+\mathrm{H}^{2}\right)}\right)$ reage com $\mathrm{O}_{2}-$ para formar $\mathrm{O}_{2}$, ficando na forma reduzida $\left(\mathrm{M}^{2+}\right)$ (Eq 1). No segundo passo da reacção de dismutação, a enzima reduzida reage com outro $\mathrm{O}_{2}-\mathrm{e}$ dois protões para formar $\mathrm{H}_{2} \mathrm{O}_{2}$, tornando à forma oxidada (Eq 2). Nos mamiferos existem três isoformas da SOD com diferentes características ${ }^{\text {[1 }}$ (Tabela 1). Outras classes de SOD foram já identificadas noutros organismos, nomeadamente uma SOD de niquel no Streptomyces sp. e em algumas cianobactérias, e SODs contendo ferro $\mathrm{em}$ bactérias, algas, tripanossomas e plantas superiores. ${ }^{[2.4)}$

Table 1 - Types of SOD enzymes in mammals $[2,4,7]$.

Tabela 1 - Tipos de isoenzimas SOD presentes nos mamiferos $[2,4,7]$.

\begin{tabular}{|c|c|c|c|}
\hline & SOD1 & SOD2 & SOD3 (EC-SOD) \\
\hline $\begin{array}{l}\text { Active centre } \\
\text { Centro activo }\end{array}$ & $\mathrm{Cu}(\mathrm{II})(\mathrm{I})+\mathrm{Zn}(\mathrm{II})$ & $\mathrm{Mn}(\mathrm{III})(\mathrm{II})$ & $\mathrm{Cr}(\mathrm{II}) / \mathrm{I})+\mathrm{Zn}$ (II) \\
\hline $\begin{array}{l}\text { Protein Structure } \\
\text { Estrutura da proteina }\end{array}$ & $\begin{array}{l}\text { Hamodimer } \\
\text { Homodimero }\end{array}$ & $\begin{array}{l}\text { Homotetramer } \\
\text { Homotetràmero }\end{array}$ & $\begin{array}{l}\text { Tetrameric glycoprotein } \\
\text { Glicoproteina tetramérica }\end{array}$ \\
\hline $\begin{array}{l}\text { Genetic locus } \\
\text { Locus genético }\end{array}$ & $21 \mathrm{q} 22.1$ & $6 q 25,3$ & $4 \mathrm{p} 15.3-\mathrm{p} 15.1$ \\
\hline Lacation & $\begin{array}{l}\text { Cytasol, nucleus and } \\
\text { mitochondrial inter } \\
\text { membrane space - }\end{array}$ & $\begin{array}{l}\text { Mitochondria } \\
\text { matrix }\end{array}$ & $\begin{array}{c}\text { Extracellular } \\
\text { compartments (plasma, } \\
\text { bymph, synovial fluid) }\end{array}$ \\
\hline Localização & $\begin{array}{l}\text { Citosol, núcleo e } \\
\text { espaço } \\
\text { intermembranat } \\
\text { mitocondrial }\end{array}$ & $\begin{array}{c}\text { Matriz } \\
\text { mitocondrial }\end{array}$ & $\begin{array}{l}\text { Compartimentos } \\
\text { extracelulares (plasma, } \\
\text { linfa, liquido sinovial) }\end{array}$ \\
\hline
\end{tabular}

\section{Catalase}

CAT enzymes are present in the peroxisomes of most aerobes, and catalyze the direct decomposition of $\mathrm{H} 2 \mathrm{O} 2$ to ground-state $\mathrm{O} 2$ and $\mathrm{H} 2 \mathrm{O}$. In animals, CAT is present in all organs and it's mostly concentrated in the liver, kidney and erythrocytes. Human CAT is a tetrameric hacmin-enzyme consisting of four identical subunits of $60 \mathrm{kDa}$, each containing $\mathrm{Fe}$ (III)-hacm at its active site . Its gene is located in chromosome 11 , band $\mathrm{p} 13$. In the $\mathrm{H} 2 \mathrm{O} 2$ dismutation, $\mathrm{H} 2 \mathrm{O} 2$ oxidizes the haem iron of the resting CAT to form an oxyferryl group with a $\pi$ cationic porphyrin radical, termed Compound I (Eq 3 ). This step is followed by oxidation of a second molecule

\section{Catalase}

As enzimas CAT estão presentes nos peroxissomas da maioria dos aeróbios e catalizam directamente a decomposição de $\mathrm{H}_{2} \mathrm{O}_{2} \mathrm{em} \mathrm{O}_{2} \mathrm{e} \mathrm{H}_{2} \mathrm{O}^{21}$. Nos animais, a CAT estă presente em todos os orgãos, encontrando-se mais concentrada no figado, rins e eritrócitos ${ }^{[2.15}$. A CAT humana é uma hemoproteina tetramérica com quatro subunidades idênticas de $60 \mathrm{kDa}$, cada uma com um grupo $\mathrm{Fe}$ (III)-hemo no seu centro activo ${ }^{[2,4,19]}$. $\mathrm{O}$ gene que codifica esta enzima localiza-se no cromossoma 11, banda p13 ${ }^{\text {!n! }}$. Na dismutação de $\mathrm{H}_{2} \mathrm{O}_{2}$, - $\mathrm{H}_{2} \mathrm{O}_{2}$ oxida o ferro(III)-hemo da CAT para formar um 
of $\mathrm{H} 2 \mathrm{O} 2$ by Compound $\mathrm{I}(\mathrm{Eq} 4)$. The catalytic rate of mammalian CAT is among the highest known enzymatic rates, and it is simply proportional to $\mathrm{H} 2 \mathrm{O} 2$ concentration over a wide range of concentrations . radical catiónico oxo-ferril-porfirínico designado de Composto I (Eq 3). Seguidamente, ocorre oxidação de uma segunda molécula de $\mathrm{H}_{2} \mathrm{O}_{2}$ pelo Composto I $(\mathrm{Eq}$ 4) ${ }^{[\text {tie }}$. A actividade catalítica da CAT está entre as maiores taxas de actividade enzimática conhecidas, sendo proporcional à concentração de $\mathrm{H} 2 \mathrm{O} 2$ para uma vasta gama de concentraçōes ${ }^{10}$.

$$
\begin{aligned}
& \text { CAT }\left(\mathrm{Por}-\mathrm{Fe}^{\mathrm{III}}\right)+\mathrm{H}_{2} \mathrm{O}_{2} \rightarrow \text { Compound I }\left(\mathrm{Po} \text { - }-\mathrm{Fe}^{\mathrm{IV}}=\mathrm{O}\right)+\mathrm{H}_{2} \mathrm{O} \\
& \text { Compound I (Por } \left.{ }^{+}-\mathrm{Fe}^{\mathrm{IV}}=\mathrm{O}\right)+\mathrm{H}_{2} \mathrm{O}_{2} \rightarrow \mathrm{CAT}\left(\mathrm{Por}-\mathrm{Fe}^{\mathrm{III}}\right)+\mathrm{H}_{2} \mathrm{O}+\mathrm{O}_{2}
\end{aligned}
$$

Net reaction/ Reacção Global : $2 \mathrm{H}_{2} \mathrm{O}_{2} \rightarrow 2 \mathrm{H}_{2} \mathrm{O}+\mathrm{O}_{2}$

\section{Glutathione peroxidases}

Glutathione peroxidases are selenium-containing enzymes that degrade a variety of peroxides, namely $\mathrm{H} 2 \mathrm{O} 2$ (Eq 5) and $\mathrm{ROOH}$ (Eq 6), by coupling their reduction with the oxidation of reduced glutathione (GSH) ,

\section{Glutationo peroxidases}

As glutationo peroxidases são seleno-enzimas capazes de degradar vários peróxidos, nomeadamente $\mathrm{H} 2 \mathrm{O} 2$ $(\mathrm{Eq}$ 5) e ROOH (Eq 6), por acoplarem a sua redução à oxidaçāo do glutationo $(\mathrm{GSH})^{12,4}$.

$$
\begin{aligned}
& 2 \mathrm{H}_{2} \mathrm{O}_{2}+2 \mathrm{GSH} \rightarrow \mathrm{GSSG}+2 \mathrm{H}_{2} \mathrm{O} \\
& \mathrm{ROOH}+2 \mathrm{GSH} \rightarrow \mathrm{GSSG}+\mathrm{H}_{2} \mathrm{O}+\mathrm{ROH}
\end{aligned}
$$

Gpx enzymes are widely distributed in animal tissues . Four isoenzymes have been identified in humans, each isoform depending on the tissue type. The classical GPx (GPx1) is cytosolic. GPx2 is an isoform present in the gastro-intestinal tract. In plasma and other extracellular fluids, a different isoenzyme (GPx3) is found. The fourth type is the phospholipid hydroperoxide glutathione peroxidase (PHGPx or GPx4), which has the ability to act upon peroxidized fatty acid residues within membranes and lipoproteins . GPx4 is a monomer, while GPx1, GPx2 and GPx 3 are tetramers. Each protein unit contains a selenium atom in the active site, in the form of selenocysteine. The catalytic activity of GPx is depicted in Fig. 4. During GPx catalysis, the selenol form of the enzyme is oxidized to selenenic acid by peroxides. This form reacts with reduced glutathione (GSH) to give $\mathrm{H} 2 \mathrm{O}$ and a selenosulphide adduct. A second GSH molecule then regenerates the enzyme's active form by attacking the selenosulphide to form oxidized glutathione (GSSG) .
As enzimas GPx estão amplamente distribuidas nos tecidos animais ${ }^{[2]}$. Nos humanos, estão identificadas quatro isoenzimas, sendo o teor de cada isoforma dependente do tipo de tecido ${ }^{[2 / 2]}$. A GPx clássica (GPx1) tem localização citosólica. A GPX2 é uma isoforma presente no trato gastro-intestinal. No plasma e fluidos extracelulares encontra-se a GPx3. O quarto tipo (GPx4) é a fosfolípido-hidroperóxido glutationo peroxidase, que actua nos residuos de ácidos gordos peroxidados de membranas e lipoproteinas ${ }^{[2]}$. A GPx4 é um monómero, enquanto que a GPx $1, \mathrm{GPx} 2 \mathrm{e} \mathrm{GPx} 3$ são tetrâmeros. Cada proteina contém um átomo de selénio no seu centro activo, na forma de selenocisteina. A actividade catalítica da GPx está esquematizada na Fig. 4. Durante a catálise, a forma selenol da enzima é oxidada a ácido selenénico pelos peróxidos. Esta forma reage com o GSH originando $\mathrm{H} 2 \mathrm{O}$ e um aducto selenossulfureto. Uma segunda molécula de GSH vai reagir com o selenossulfureto formando glutationo oxidado (GSSG) e regenerando a forma activa da enzima $^{[2,1]}$. 


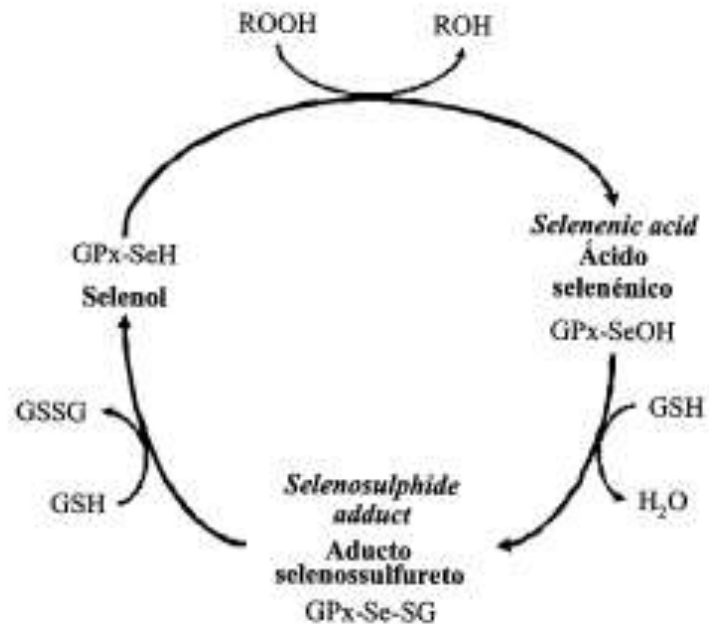

Figure 4-Catalytic cycle of glutathione peroxidase (adapted from ${ }^{(17)}$ ).

Figura 4 - Ciclo catalitico da glutationo peroxidase (adaptado de ${ }^{[17}$ ).

The catalytic activity of GPx involves the oxidation of GSH in GSSG. However, under physiological conditions, the cellular proportion GSH/GSSG is high, because GSSG is reduced back to GSH by glutathione reductase (GR). The catalytic activity of this enzyme requires NADPH, according to the following reaction (Eq 7):

$$
\mathrm{GSSG}+\mathrm{NADPH}+\mathrm{H}^{*} \rightarrow 2 \mathrm{GSH}+\mathrm{NADP}^{*}
$$

A actividade catalitica da GPx envolve assim a oxidação de GSH a GSSG. No entanto, em condições fisiológicas, a razão GSH/GSSG nas células é elevada, pois o GSSG é reduzido a GSH pela glutationo reductase $(\mathrm{GR})^{1 ., 2]}$. A actividade catalitica da GR requer $\mathrm{NADPH}$, de acordo com a seguinte reacção (Eq 7):
Cellular NADPH can be provided by different sources, including glucose-6-phosphate dehydrogenase (G-6$\mathrm{PDH})$ and NADP+-dependent isocitrate dehydrogenase (ICD) (Fig. 3).

Besides being a cofactor for GPx enzymes, GSH plays other important antioxidant roles. This ubiquitous thiolcontaining tripeptide can directly scavenge RS, such as $\mathrm{HO}$ and 102 . Moreover, it is used by glutathione Stransferases (GSTs) to conjugate and eliminate reactive compounds, including products formed in vivo during oxidative stress. Glutathione is also able to regenerate important antioxidants back to their active forms (e.g. vitamins $\mathrm{C}$ and $\mathrm{E}$ ), being this capacity linked with the ratio GSSG/2GSH, which is determinant for the redox state of the cellular milieu. In a non-stressed cell, glutathione is present in the cytosol at a concentration between 1 and $10 \mathrm{mM}$, being approximately $99 \%$ in the form of GSH and approximately $1 \%$ as GSSG.

\section{Oxidative damage}

While, under physiological circumstances, ROS levels are kept under tight control by endogenous antioxidants , in oxidative stress ROS production overwhelms the
O NADPH celular pode ser proveniente de diferentes fontes, incluindo a glucose-6-fosfato desidrogenase (G-6-PDH) e a isocitrato desidrogenase NADP+dependente (ICD) (Fig. 3) ${ }^{[2]}$.

Para além de ser um cofactor da GPx, o GSH desempenha outros papeis antioxidantes importantes. Este tripéptido tiol ubiquitário pode sequestar espécies reactivas directamente, nomeadamente $\mathrm{HO} \mathrm{e}^{1} \mathrm{O}_{2}{ }^{[8,18]} \cdot \mathrm{O}$ GSH é também usado pelas glutationo-S-transferases (GSTs) para conjugar e eliminar compostos reactivos, incluindo produtos formados in vivo em situações de stress oxidativo ${ }^{(1,2,8,14)}, \mathrm{O}$ glutationo tem também um papel importante na regeneração de outros antioxidantes na sua forma activa (ex, vitaminas $\mathrm{CeE}$ ), estando esta capacidade relacionada com a proporção GSSG/2GSH ${ }^{10}$, a qual é determinante para o estado redox do ambiente celular. Em células não sujeitas a stress oxidativo, a concentração citosólica de glutationo varia entre 1 e $10 \mathrm{mM}$, estando cerca de $99 \%$ na forma de GSHe cerca de $1 \%$ como GSSG ${ }^{[2,1 x]}$.

\section{Lesões oxidativas}

Enquanto que em situações fisiológicas os niveis de ERO são mantidos sob controlo rigoroso pelos antioxidantes 
detoxification capacities. This imbalance will induce different cellular responses, depending on the cell type end the severity of oxidative stress. Many cells respond to mild oxidative stress by proliferating. As oxidative stress increases, cells may also up-regulate their antioxidant defence systems. However, higher oxidative stress levels may lead to damage in biomolecules such as DNA, proteins, lipids, or carbohydrates, resulting in oxidative cell injury. Cells may recover from this damage by displacing the damaged molecules, or repairing them (e.g. DNA), or they can survive with persistent lesions. In case of intense oxidative stress, cells may even become senescent, i.e., they survive but are no longer able to divide. Under highly oxidizing conditions, mechanisms of cell death (apoptosis and necrosis) often occur.

Beyond the direct oxidation of biomolecules, ROS deleterious effects may be mediated by other mechanisms, including enzyme, neurotransmitters and hormone degradation, and the iron release from storage sites and consequent reaction with $\mathrm{H} 2 \mathrm{O} 2$ producing $\mathrm{HO}$ radicals . Furthermore, ROS and specially $\mathrm{O} 2$ - have been shown to promote different pro-inflammatory actions such as endothelial cell damage, and increased microvascular permeability, chemotactic factors formation, neutrophils recruitment at inflammation sites, and activation of polyADP-ribose polymerase . By promoting tissue injury and inflammation phenomena, ROS are involved in many pathological conditions as exemplified in Table 2. Additionally, ROS play an important role in cancer, being involved in the cell transformation, metastasis and angiogenesis processes . endógenos ${ }^{\text {(s) }}$,em situaçōes de stress oxidativo a produçāo de ERO ultrapassa as capacidades de destoxificaçăo. Este desiquilibrio vai induzir diferentes respostas celulares, dependendo do tipo de células e do nível de stress oxidativo. Muitas células respondem ao stress oxidativo ligeiro aumentando a proliferação celular. Quando o stress oxidativo aumenta, as células podem ainda estimular os seus sistemas de defesa antioxidante. No entanto, niveis de stress oxidativo mais intensos podem levar à oxidação de biomoléculas como o ADN, proteinas, lipidos ou glúcidos, resultando em lesões celulares oxidativas. As células podem recuperar substituindo, ou reparando (ex. ADN), as moléculas lesadas, ou podem sobreviver com lesరెes persistentes. Em situações de elevado stress oxidativo as células podem ainda tornar-se senescentes, i.e., sobreviverem mas sem capacidade de se dividirem, ou podem mesmo ocorrer mecanismos de morte celular (ex. apoptose e necrose) ${ }^{[2]}$.

Para além da oxidação directa de biomolèculas, os efeitos deletérios das ERO podem ser mediados por outros mecanismos, incluindo a degradação de enzimas, neurotransmissores $\mathrm{e}$ hormonas, e a libertaçåo de ferro dos seus locais de armazenamento e consequente reacção com $\mathrm{H}^{2} \mathrm{O}^{2}$ produzindo radicais $\mathrm{HO}^{\left[2,4, \pi^{3}\right]}$. Para além disso, as EROe sobretudo o $\mathrm{O}^{2-}$ têm demonstrado promover diversas acçōes pro-inflamatórias tais como lesão endotelial, aumento da permeabilidade microvascular, formação de factores quimiotácticos, recrutamento de neutrófilos para os locais de inflamação e activação da poli-ADP-ribose polimerase ${ }^{16.7}$. Ao promover fenómenos de lesão tecidular e inflamaçāo, as ERO estão envolvidas em muitas condições patológicas ${ }^{[i, 7,19]}$, tal como exemplificado na Tabela 2. Adicionalmente, as ERO desempenham um papel importante no cancro, entando implicadas nos processos de transformação, metastização e angiogénese ${ }^{(t) \text { ? }}$.

Table 2 - Examples of elinical conditions in which the involvement of ROS has been suggested ${ }^{[0,7}, 19 ;$ Tabela 2- Exemplos de condiçōes clinicas nas quais o envolvimento de ERO tem sido sugerido ${ }^{\mid 6,2,19 !}$

\begin{tabular}{|c|c|}
\hline $\begin{array}{l}\text { Category } \\
\text { Categoria }\end{array}$ & $\begin{array}{l}\text { Examples } \\
\text { Exemplos }\end{array}$ \\
\hline Inflammatory conditions & $\begin{array}{l}\text { Pain, Crohn's disease, rheumatoid arthritis, asteoarthritis, dermatitis, } \\
\text { psariasis }\end{array}$ \\
\hline Situaçōes inflamatórias & Dor, doença de Crohn, artrite reumatoide, osteoartrite, dermatite, psoriase \\
\hline Cardiovascular diseases & Ischemia-reperfusion injury, shock, atherosclenasis \\
\hline Doenças cardiovasculares & Isquémia-reperfusão, choque, aterosclerose \\
\hline Doenças neurodegenerativas & Doença de Parkinson, Alzheimer, esclerose lateral amiotrófica \\
\hline Neurodegenerative diseases & Parkinson's, Alzheimer, amiotrophic laternal sclerosis \\
\hline Pulmonary diseases & Asthma, chronic obstructive pulmonary disease \\
\hline Docnças pulmonares & Asma, doença pulmonar obstrutiva crónica \\
\hline Oncology & Side effects of chemo-and radiotherapy \\
\hline Oncologia & Efeitos secundários da quimio-e radioterapia \\
\hline Reproductive system & Erectile dysfunction, infertility \\
\hline Sistema reprodutor & Disfunção erectil, infertilidade \\
\hline
\end{tabular}


In addition to the mentioned pathologies, ROS are of utmost importance in Toxicology, since oxidative damage is a relevant mechanism of action of many toxicants. In fact, exposure to a number of xenobiotics may result in the ROS generation and/or release. This can occur directly or upon metabolization, redox reactions, redox cycling processes, via lipid peroxidation, or by stimulation of endogenous ROS production. As abovementioned, among the xenobiotics whose effects seem to be, at least partially, ROS-mediated some pesticides (e.g. paraquat, rotenone and pentachlorophenol), metals (e.g. vanadium, cadmium, mercury, iron, arsenic chromium), and drugs (e.g. bleomycin, gentamicin, anthracyclines and rifamycin) are found .

\section{Conclusion}

The ROS involvement in numerous pathological processes justifies the widespread interest in antioxidants. If endogenous antioxidant defences are overwhelmed, the use of exogenous compounds to remove excess ROS may provide a promising strategy to prevent and treat a variety of diseases and intoxication conditions. This supports the use of antioxidants in many foods and supplements. Furthermore, many attempts towards the use of synthetic antioxidants as potential drugs have been developed. Although some of these strategies have shown convincing results, the complexity of the effects mediated by ROS and the redox-modulated cellular pathways, precludes a straightforward intervention. Many issues still need to be clarified in order to propose effective redox-based therapeutic strategies.

\section{Acknowledgements}

A.S. Fernandes acknowledges FCTS (Fundação para a Ciência e a Tecnologia)- Portugal for the financial support (Post-doc grant SFRH/BPD/69042/2010).
Para além das patologias mencionadas, as ERO são de grande importância em Toxicologia dado que as lesర̃es oxidativas são um mecanismo de toxicidade relevante para o modo de acção de muitos agentes tóxicos. De facto, a exposição a muitos xenobióticos pode resultar na libertação ou geração de ERO, o que poderá ocorrer directamente ou após metabolização, reacçōes redox, peroxidaçăo lipídica ou por estimulação da produçāo endógena de ERO. Como acima mencionado, entre os xenobióticos cujos efeitos são, pelo menos parcialmente, atribuidos a ERO estão alguns pesticidas (ex. paraquato, rotenona e pentaclorofenol), metais (ex. vanádio, cádmio, mercúrio, ferro, arsénio, crómio) e färmacos (ex. bleomicina, gentamicina, antraciclinas e rifamicina) ${ }^{12 \cdot 29}$.

\section{Conclusão}

O envolvimento das ERO em muitos fenómenos patológicos justifica o interesse generalizado pelos antioxidantes. Quando as defesas antioxidantes endógenas não sâo suficientes, a utilização de compostos exógenos para remoçăo das ERO em excesso pode constituir uma estratégia promissora para prevenir e tratar várias doenças e intoxicações. Esta ideia suporta a utilização de antioxidantes em muitos alimentos e suplementos. Para além disso, vários esforços têm sido feitos no sentido de obter antioxidantes sintéticos como potenciais fărmacos ${ }^{|2-2 \cdot 2| \mid}$. Apesar de algumas destas estratégias terem mostrado resultados relevantes, a complexidade de efeitos mediados por ERO e as várias vias celulares susceptiveis de modulação redox excluem a possibilidade de uma intervenção simplista. Muitas questôes têm ainda que ser clarificadas de modo a que se possam vir a propor estratégias de terapêutica redox eficazes.

\section{Agradecimentos}

A.S. Femandes agradece $o$ apoio financeiro da Fundação para a Ciência e a Tecnologia, Portugal (bolsa post-doc SFRH/BPD/69042/2010).
[1] Sies H. Oxidative stress: oxidants and antiaxidants. Exp Physiol 1997;82:291-5.

[2]. Halliwell B, Gutteridge JMC. Free Radicals in Biology and Medicine. New York: Oxford University Press. 2007

[3]. Halliwell B. Biosbcrnistry of oxidative stress. Bjochem Soc Trans 2007;35:1147-50.

[4]. Mates M. Effects of antioxidant enzymes in the molectalar control of reactive axygen species toxicology. Toxicology 2000;153;83-104.

[5]. Finkei T, Holbrook NJ. Oxidants, oxidative stress and the biology of ageing. Nature $2000,408: 239.47$.
[6]. Salvemini D, Muscoli C, Riley DP, CuzzocreaS, Superoxide dismurase mimetics. Pulm Pharmacol Ther 2002;15:439-47

[7]. Muscoli C, Cuzzocrea S, Riley DP, Zweier IL, Thiemermann C, Wang ZQ, et al. On the selectivity of superoxide dismulase mimetics and its importanet in pharmacologieal studies. Br J Pharmacol 2003; 140:445-60.

[8]. Droge W. Free radicals in the physiological control of cell function. Physiol Rev 2002;82:47:95.

[9]. Valko M, Leibfritz. D, Moncol J, Cronin MT, Mavur M, Telser J. Free radicals and antioxidants in sormal physiological functions and buman disease,
Int J Biochem Cell Biol 2007:39:44-84.

[10]. Day BJ. Catalytic antioxidants: a radical approsech to new therapeutics. Drug Discov Todsy 2004-9:557-66.

[11]. Halliweil B, Whiveanan M. Measuring reactive species and oxidative damage in vivo and in cell culture: how should you do it and what do tbe results mean? Br J Pharmicol 2004:142:231-55.

[12]. Mates JM, Perez-Gamez C, Nunez de Castro I Antioxidant enaymes and human distases. Clin Bioctem 1999;32:595-603.

[13]. Oberley LW. Therapeutic Manipalation of the Intracellular Redox State. American Association for 
Cancer Research Edocation Book 2006:255-8. [14]. MoCord JM, Edeas MA. SOD, oxidative stres: and human pathologies: a brief history and a fiature vision. Biomed Pharmacocher 2005-59-139-42.

[IS]. Quan F, Komeluk RG, Tropak MB, Gravel RA. Isolation and characterization of the haman catalase gene. Nucleic Acids Res 1986;14 5321-35:

[16). Kirkman HN, Gactsai GF. Mammalian catalase: a venerable enzyme with new mysteries. Trends Biochem 8ci 2007;32:44-50.

[17]. Mugesb G, Singh HB. Synthetic crganoselenium compounds as antioxidants glutathione peroxidase sctivity. Oocen Soc Rev $2000 ; 29: 347-57$

[18]. Pastore A, Federici G, Bertini E, Piemoese F. houlysis of glutathioes: implication in redox and detoxification. Clin Chimasta 2003;331:19-39.

[19]. Aganwal A, Nandipati KC, Sharma RK, Zippe

$C D$, Raina $R$. Role of oxidative stress in the pathophysiological mechanism of erectile dysfunction. J Androl 2006;27:335-47.

[20]. Cuzzoctea S, Mazzon E, Dugo L, Serraino L, D Paola R, Britti D, et al. A role for superoxide in gentamicin-mediated nephropactiy in rats, Eur J Pharmacol 2002;450:67-76.

[21]. Batinic-Haberle I, Rebougas JS, Spasojevi? I. Superoxide dismatase mimics: chemistry, pbarmacology and therapeutic potential. Antiox Redox Signal 2010;13-877-918.

[22]. Fernandes AS, Gaspar J. Cabral MF, Caneiras C. Guedes R, Rueff J, et al. Macrocyelic coppet(II) complexes: superoxide scayenging activity, structural studies and cytotoxicity evaluation. J Inoce Biochem 2007; 101:849-58.

[23]. Femandes AS, Gaspar J, Cabnul MF, Rueff J Castro M. Batinic-Haberle I, er al. Protective role of ortbo-substinated Mr(III) N-slkylpyridylporpbyrins against the oxidstive injury induced by tertbutylhydropercoxide. Free Radic Res 2010;44:43040.

[24]. Fermandes AS, Sercjo J, Gaspar J, Cabral F, Bettencourt AF, Rueff J, et al. Oxidative injury in V79 Chinese hamster eells: protective role of the - superoxide dismutase mimetic MnTM-4-PyP. Cell Bial Toxicol 2010;26:91-101. 